\title{
Globaltest and GOEAST: two different approaches for Gene Ontology analysis
}

\author{
Ina Hulsegge*, Arun Kommadath and Mari A Smits
}

\author{
Address: Animal Breeding and Genomics Centre, Animal Sciences Group Wageningen UR, P.O. Box 65, 8200 AB Lelystad, The Netherlands \\ Email: Ina Hulsegge* - ina.hulsegge@wur.nl; Arun Kommadath - arun.kommadath@wur.nl; Mari A Smits - mari.smits@wur.nl \\ * Corresponding author
}

from EADGENE and SABRE Post-analyses Workshop

Lelystad, The Netherlands. 12-14 November 2008

Published: 16 July 2009

BMC Proceedings 2009, 3(Suppl 4):SI0 doi:I0.II86/I753-656I-3-S4-SI0

This article is available from: http://www.biomedcentral.com/I753-656I/3/S4/SIO

(c) 2009 Hulsegge et al; licensee BioMed Central Ltd.

This is an open access article distributed under the terms of the Creative Commons Attribution License (http://creativecommons.org/licenses/by/2.0), which permits unrestricted use, distribution, and reproduction in any medium, provided the original work is properly cited.

\begin{abstract}
Background: Gene set analysis is a commonly used method for analysing microarray data by considering groups of functionally related genes instead of individual genes. Here we present the use of two gene set analysis approaches: Globaltest and GOEAST.

Globaltest is a method for testing whether sets of genes are significantly associated with a variable of interest. GOEAST is a freely accessible web-based tool to test GO term enrichment within given gene sets. The two approaches were applied in the analysis of gene lists obtained from three different contrasts in a microarray experiment conducted to study the host reactions in broilers following Eimeria infection.

Results: The Globaltest identified significantly associated gene sets in one of the three contrasts made in the microarray experiment whereas the functional analysis of the differentially expressed genes using GOEAST revealed enriched GO terms in all three contrasts.

Conclusion: Globaltest and GOEAST gave different results, probably due to the different algorithms and the different criteria used for evaluating the significance of GO terms.
\end{abstract}

\section{Background}

Several methods have recently been developed for gene set analysis of microarray data $[1,2]$. These methods evaluate differential gene expression patterns of groups of functionally related genes instead of individual genes. The aim is to discover gene sets whose expression patterns are associated with phenotypes of interest. Genes can be grouped together into gene sets, for example, based on function (Kyoto Encyclopedia of Genes and Genomes (KEGG), Gene Ontology (GO) [3]) or location (chromo- some, cytoband). In this paper we present the results obtained with two different gene set analysis approaches: Globaltest [4] and Gene Ontology Enrichment Analysis Software Toolkit (GOEAST) [5]. Globaltest is a method for testing whether sets of genes are significantly associated with a variable of interest. The method is based on a prediction model for predicting a response variable from the gene expression measurements of a set of genes. The null hypothesis tested is that expression profile of the genes in the gene set is not associated with the response 
variable. GOEAST is a freely accessible web-based tool to test GO term enrichment within given gene sets. It supports the analysis of data from common commercial microarray platforms and even customized arrays if the probe annotation file in the required format is provided.

These approaches were applied in the analysis of gene lists obtained from three different contrasts in a microarray experiment conducted to study the host reactions in broilers following Eimeria infection.

\section{Methods}

Globaltest

The Globaltest allows different kinds of variables to be tested, based on which it determines the correct model (logistic, linear or survival).

The Globaltest calculates the p-value using different methods, the most important ones being permutations and the asymptotic distribution. Here the asymptotic distribution was used. All p-values were corrected for multiple testing using Benjamini and Hochberg's False Discovery Rate (FDR) [6]. GO terms were considered significant if the pvalue after correcting for multiple testing, was below 0.05 . The influence of individual genes in a GO term was evaluated using z-score calculated in Globaltest. Genes with zscores that are greater than 2 were considered significant contributors to the GO term. GO terms which matched only one gene were excluded from the analysis.
The Globaltest package also offers plots to visualize the effects of different genes and different samples on the test result: 1 . Sample plot: how good a sample fits to its phenotype, 2. Checkerboard: correlation between samples, and 3. Gene plot: Influence of individual genes to test statistics.

$\mathrm{R}$ version 2.8.0 was used to run the Globaltest package (version 4.12.0).

\section{Availability}

Globaltest: http://www.bioconductor.org/packages/2.0/ bioc/html/globaltest.html

\section{R: http://www.r-project.org/}

\section{GOEAST}

For GOEAST all GO terms with less than 5 probes associated with it on the array are discarded from the test because the statistical analysis would not be appropriate then.

The Fisher's exact test available in GOEAST was used separately on the 2-fold upregulated and downregulated gene lists for each of the three contrasts. The $\mathrm{p}$-values were adjusted using Benjamini-Yekutieli method [7] with cutoff for FDR control set at 0.1. The Benjamini-Yekutieli method is more suitable for positively related multiple tests as is the case for enriched GO terms within gene lists

Table I: Top 5 GO terms in contrast MM8-PM8 identified by Globaltest

\begin{tabular}{|c|c|c|c|c|c|}
\hline GO term ID & GO term description & Number of genes in GO term ${ }^{a}$ & Number of genes affected b & $\mathrm{P}$-value & FDR adjusted $p$-value ${ }^{c}$ \\
\hline \multicolumn{6}{|c|}{ Biological Process } \\
\hline GO:0051017 & actin filament bundle formation & 6 & 2 & 0.002 & 0.047 \\
\hline GO:0006996 & $\begin{array}{l}\text { organelle organization and } \\
\text { biogenesis }\end{array}$ & 3 & 2 & 0.002 & 0.047 \\
\hline GO:0015816 & glycine transport & 2 & I & 0.003 & 0.047 \\
\hline GO:0016042 & lipid catabolic process & 7 & 6 & 0.003 & 0.047 \\
\hline GO:0009113 & purine base biosynthetic proces & 4 & 2 & 0.003 & 0.047 \\
\hline \multicolumn{6}{|c|}{ Molecular function } \\
\hline GO:0019976 & interleukin-2 binding & 2 & 2 & 0.002 & 0.040 \\
\hline GO:0015187 & $\begin{array}{l}\text { glycine transmembrane transporter } \\
\text { activity }\end{array}$ & 2 & 1 & 0.003 & 0.040 \\
\hline GO:0031013 & troponin I binding & 2 & 1 & 0.003 & 0.040 \\
\hline GO:0003847 & $\begin{array}{l}\text { I-alky|-2- } \\
\text { acetylglycerophosphocholine.... }\end{array}$ & 2 & 2 & 0.003 & 0.040 \\
\hline GO:0004438 & $\begin{array}{l}\text { phosphatidylinositol-3-phosphatase } \\
\text { activity }\end{array}$ & 2 & 2 & 0.003 & 0.040 \\
\hline \multicolumn{6}{|c|}{ Cellular component } \\
\hline GO:0001726 & ruffle & 18 & 9 & 0.003 & 0.034 \\
\hline GO:0005719 & nuclear euchromatin & 2 & I & 0.003 & 0.034 \\
\hline GO:0005884 & actin filament & 12 & 6 & 0.003 & 0.034 \\
\hline GO:0000307 & cyclin-dependent protein kinase... & 5 & 3 & 0.004 & 0.034 \\
\hline GO:0016529 & sarcoplasmic reticulum & 4 & 3 & 0.004 & 0.034 \\
\hline
\end{tabular}

a Number of genes within each GO term.

$b$ Genes differentially expressed with z-score $>2.0$

c The $\mathrm{p}$-values were adjusted for multiple hypotheses testing with Benjamini and Hochberg to control the false discovery rate (FDR). 
[5]. To reduce the FDRs caused by over-representation of neighbouring GO terms due to their hierarchical dependency, Adrian Alexa's improved weighted scoring algorithm [8] which is implemented in GOEAST was used.

The results from GOEAST analysis are presented in 3 ways: an HTML table providing detailed information of enriched GO terms and their associated genes; a plain-text file of enriched GO terms; and separate graphical output files showing the hierarchical relationships of enriched GO terms in the $3 \mathrm{GO}$ categories.

Besides the Fisher's exact test, GOEAST also supports hypergeometric test and $\chi^{2}$-test as well as other methods for multiple testing correction (Hochberg, Bonferroni, Hommel).

Availability

http://omicslab.genetics.ac.cn/GOEAST/

\section{Results}

Globaltest

The Globaltest takes into account the entire raw expression data. The overall gene expression profile for the three contrasts (MM8-PM8, MM8-MA8 and MM8-MM24) was significantly associated $(\mathrm{p}<0.05)$ with their outcomes, the p-values using the asymptotic method being 0.006 , 0.032 and 0.021 respectively. This shows that the overall gene expression pattern of MM8 chicken differs significantly from that of PM8, MA8 and MM24 chicken. Therefore there is a potential in predicting infection from gene expression data.

GO terms (biological process, molecular function, cellular component) were used for gene set analysis. After correction for multiple testing, no significant gene sets (GO terms) were found in MM8-MA8 and MM8-MM24 contrasts. However, in the MM8-PM8 contrast, 527, 331 and 180 out of a total of 1679,838 and 336 GO-terms, were found to be significant $(\mathrm{p}<0.05)$ for biological process, molecular function and cellular component, respectively. The five most significant terms for each GO category are listed in Table 1. The influence of individual genes on the results for the GO term "ruffle" is shown in Figure 1. Nine

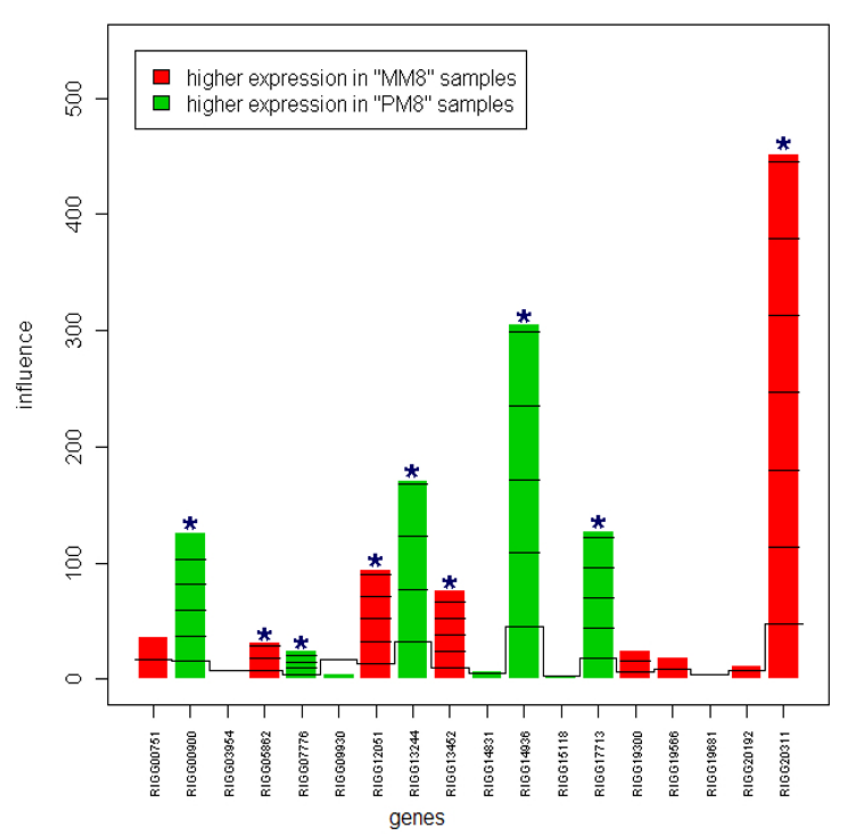

Figure I

Geneplot of the GO term "ruffle". The gene plot shows a bar and a reference line for each gene tested. The reference line reflects the expected influence if the gene was not associated with the GO term "ruffle". In case the height of the bar exceeds the reference line, the gene significantly influences the GO term "ruffle". Marks indicate the standard deviations by which the bar exceeds the reference line. The bars are coloured to indicate a higher expression in PM8 compared to MM8 (green) or a higher expression in MM8 compared to PM8 (red). * Genes clearly above the reference.

genes were clearly above the reference line and nine genes did not show an effect.

\section{GOEAST}

In GOEAST, only differentially expressed genes over 2 fold level within the gene lists from each of the three contrasts were taken into account. The analysis for enriched GO terms by the Fisher's exact test revealed a large number of enriched GO terms at 0.1 FDR level for all three contrasts (Table 2). GOEAST identified 34, 12 and 39 enriched GO

Table 2: Summary of results of Fisher's exact test in GOEAST for the 3 contrasts

\begin{tabular}{llrrr}
\hline Contrast & $\begin{array}{l}\text { Number of significantly expressed genes } \\
(>2 \text { fold up/down regulation) }\end{array}$ & $\begin{array}{l}\text { Number of genes with GO } \\
\text { annotation }\end{array}$ & $\begin{array}{l}\text { Number of enriched GO terms } \\
\text { (adjusted } p \text {-value<0.1) }\end{array}$ \\
\hline MM8-PM8 & up & 659 & 303 & 34 \\
& down & 647 & 216 & 35 \\
MM8-MA8 & up & 22 & 9 & 12 \\
& down & 57 & 13 & 31 \\
MM8-MM24 & up & 131 & 58 & 39 \\
& down & 515 & 146 & 57
\end{tabular}


terms at FDR 0.1 level for the up regulated genes in the three contrasts MM8-PM8, MM8-MA8 and MM8-MM24 respectively, whereas for the down regulated genes, 35, 31 and 57 enriched GO terms were identified in the three contrasts respectively. A relatively high number of enriched GO terms were found within the small number of differentially expressed genes in the contrast MM8-MA8 (Table 2). This is probably due to the fact that many of the enriched GO terms consisted of just 1 or 2 well annotated genes. For example, several GO terms consisted of the gene TICAM1 alone. The top 5 GO terms within each GO category that were significantly enriched among differentially expressed genes for the 3 contrasts are presented in Additional file 1. The highest number of enriched GO terms were found for the GO category biological process.

\section{Discussion}

In this study, two different approaches for gene set analysis were used to analyse three contrasts made in a microarray experiment. The Globaltest is a method for testing whether sets of genes are significantly associated with a variable of interest. GOEAST, a web based software, tests for enriched GO terms in specified gene sets.

The Globaltest is a direct gene set testing method and does not start from a list of differential expressed genes, but from the raw expression data. An advantage of Globaltest compared to GOEAST is its ability to identify GO terms with genes that have limited changes in gene expression. With Globaltest, enriched GO terms can be found because only a few genes are highly differentially expressed or because many genes are only slightly differentially expressed. This may help to distinguish the key player genes of the affected GO term. The identification of genes contributing more or less to particular biological processes and molecular functions may be of great help in guiding further investigation of the pathways.

For Globaltest, given the small sample size (10 microarrays) a permutation distribution could not generate a unique p-value and therefore the asymptotic distribution was used. Although the asymptotic distribution is correct for large sample sizes, it also gives a good indication for small sample sizes [4].

From GOEAST results, it was noted that several enriched GO terms were associated with only 1 or a few genes in the tested gene lists. Though the terms still appear to be statistically significant, their biological relevance should be carefully looked into.

For example, 3 among the top 5 GO 'biological process' terms enriched in the list of down regulated genes of the contrast MM8-MA8 had one and the same gene, TICAM1, annotated to that term. However, these terms may still be biologically relevant since the TICAM1 gene is known to be involved in innate immunity against invading pathogens and therefore important in the context of the experiment that generated the gene lists.

We found different results for the two methods probably due to the different algorithms used and also the different criteria used for evaluating the significance of GO terms. Different results achieved by different gene set analysis methods were previously reported by other authors [2,9].

\section{Conclusion}

The Globaltest and GOEAST gave different results, probably due to the different algorithms and also the different criteria used for evaluating the significance of GO terms. This confirms that different gene set analysis methods perform differently and that they do not necessarily lead to the same biological conclusions. A pitfall in interpretation of the results presented here is the lack of sufficient annotation of the probes used in this microarray experiment.

\section{List of abbreviations used}

FDR: False Discovery Rate; GO: Gene Ontology; GOEAST: Gene Ontology Enrichment Analysis Software Toolkit; KEGG: Kyoto Encyclopedia of Genes and Genomes

\section{Competing interests}

The authors declare that they have no competing interests.

\section{Authors' contributions}

The three authors contributed equally to this paper.

\section{Additional material}

\section{Additional file 1 \\ Top 5 GO terms identified by GOEAST for each microarray contrast. This file presents the top $5 \mathrm{GO}$ terms within each GO category identified by Fisher's exact test in GOEAST to be significantly enriched among the $>2$ fold differentially expressed genes for the 3 contrasts: MM8-PM8, MM8-MA8 and MM8-MM24 \\ Click here for file \\ [http://www.biomedcentral.com/content/supplementary/1753- 6561-3-S4-S10-S1.doc]}

\section{Acknowledgements}

The authors wish to acknowledge Dr. J.M.J. Rebel et al. from Animal Sciences Group Wageningen UR, Lelystad, The Netherlands for providing the Chicken infection data set and EADGENE for financial support (EU Contract No. FOOD-CT-2004-5064l6).

This article has been published as part of BMC Proceedings Volume 3 Supplement 4, 2009: EADGENE and SABRE Post-analyses Workshop. The full contents of the supplement are available online at http://www.biomedcen tral.com $/|753-656| / 3$ ? issue=S4. 


\section{References}

I. Khatri P, Dr\$ghici S: Ontological analysis of gene expression data: Current tools, limitations, and open problems. Bioinformatics 2005, 2 I (18):3587-3595.

2. Song S, Black M: Microarray-based gene set analysis: a comparison of current methods. BMC Bioinformatics 2008, 9(I):502.

3. Ashburner M, Ball CA, Blake JA, Botstein D, Butler H, Cherry JM, Davis AP, Dolinski K, Dwight SS, Eppig JT, et al.: Gene ontology: Tool for the unification of biology. Nat Genet 2000, 25(I):25-29.

4. Goeman JJ, van de Geer SA Van de, De Kort F, van Houwellingen HC: A global test for groups of genes: Testing association with a clinical outcome. Bioinformatics 2004, 20(I):93-99.

5. Zheng Q, Wang XJ: GOEAST: a web-based software toolkit for Gene Ontology enrichment analysis. Nucleic Acids Res 2008, 36(Web Server issue):W358-W363.

6. Benjamini $Y$, Hochberg $Y$ : Controlling the False Discovery Rate: A Practical and Powerful Approach to Multiple Testing. Journal of the Royal Statisical Society Series B (Methodological) 1995, 57(I):289-300.

7. Benjamini $Y$, Yekutieli $D$ : The control of the false discovery rate in multiple testing under dependency. Ann Stat 200I, 29(4): I I65-II88.

8. Alexa A, Rahnenfuhrer J, Lengauer T: Improved scoring of functional groups from gene expression data by decorrelating GO graph structure. Bioinformatics 2006, 22(13): 1600-1607.

9. Dinu I, Liu Q, Potter JD, Adewale AJ, Jhangri GS, Mueller T, Einecke G, Famulsky K, Halloran P, Yasui Y: A Biological Evaluation of Six Gene Set Analysis Methods for Identification of Differentially Expressed Pathways in Microarray Data. Cancer Inform 2008, 6:357-368.

Publish with Biomed Central and every scientist can read your work free of charge

"BioMed Central will be the most significant development for disseminating the results of biomedical research in our lifetime. "

Sir Paul Nurse, Cancer Research UK

Your research papers will be:

- available free of charge to the entire biomedical community

- peer reviewed and published immediately upon acceptance

- cited in PubMed and archived on PubMed Central

- yours - you keep the copyright

Submit your manuscript here:

http://www.biomedcentral.com/info/publishing_adv.asp
BiolMedcentral 